\title{
Unspecified Type Delusional Disorder
}

National Cancer Institute

\section{Source}

National Cancer Institute. Unspecified Type Delusional Disorder. NCI Thesaurus. Code C94389.

A subtype of delusional disorder applied when the dominant delusional theme cannot be clearly determined. 